\title{
Antoine Leclerc \& compagnies. Un architecte singulier au carré
}

Pauline Nerfin

\section{(2) OpenEdition}

1 Journals

Édition électronique

URL : http://journals.openedition.org/edl/975

DOI : $10.4000 /$ edl. 975

ISSN : 2296-5084

Éditeur

Université de Lausanne

\section{Édition imprimée}

Date de publication : 15 mars 2017

Pagination : 133-152

ISBN : 978-2-940331-64-2

ISSN : 0014-2026

\section{Référence électronique}

Pauline Nerfin, «Antoine Leclerc \& compagnies. Un architecte singulier au carré », Études de lettres [En ligne], 1 | 2017, mis en ligne le 15 mars 2019, consulté le 17 décembre 2020. URL : http:// journals.openedition.org/edl/975; DOI : https://doi.org/10.4000/edl.975 


\section{ANTOINE LECLERC \& COMPAGNIES UN ARCHITECTE SINGULIER AU CARRÉ}

Après neuf ans passés dans la capitale française, le jeune architecte DPLG Antoine Leclerc (1874-1963) revient dans sa Genève natale au tournant du siècle. Dès 1900, il s'associe avec son frère, ingénieur, et un autre architecte, Gaston Le Cerf; c'est au sein de ce trio tempétueux que s'élaborent d'originaux et étonnants plans de bâtiments. Au bout de quelques années, Leclerc forme un nouveau bureau, signant des formes plus consensuelles, avec l'architecte Charles-Auguste Gambini, qui semble assurer le côté plus commercial de l'affaire. Après la Première Guerre mondiale, il collabore avec un autre architecte également formé aux beaux-arts de Paris, Alexandre Camoletti, avant de sceller sa dernière raison sociale, Leclerc \& Cie, avec sa fille, Marie-Louise, première femme architecte de Suisse romande. Par ailleurs, membre de la Fédération suisse des architectes (FAS), de la Société des ingénieurs et architectes suisses (SIA) et de l'Association Syndicale des Architectes pratiquants du Canton de Genève (ASA), il siège dans divers jurys régionaux et commissions de l'École des beaux-arts genevoise. La longue carrière d'Antoine Leclerc permet aussi d'émettre des hypothèses sur l'impact de ces coopérations sur sa production architecturale.

\section{Préambule}

Antoine Leclerc est un architecte genevois dont la carrière s'étale sur soixante-quatre ans. Né en 1874, il construit son premier bâtiment en 1899, à 25 ans, et il continue à dessiner des projets jusqu'en 1963, année de son décès. Son activité professionnelle traverse ainsi plus de la moitié $\mathrm{du} \mathrm{XX}^{\mathrm{e}}$ siècle. Ce que l'on peut donc considérer, à juste titre, comme une très longue carrière, nous offre un large terrain d'étude pour observer et analyser les mécanismes et raisons qui sous-tendent les multiples associations et collaborations scellées par Antoine Leclerc. Architecte méconnu, oublié, il est pourtant un personnage atypique, singulier; et 
révélateur sinon symptomatique de la manière dont étaient organisés les architectes de son temps.

Cet essai se présente comme un cas d'étude: autour de la figure d'Antoine Leclerc, nous allons tenter de comprendre le fonctionnement de son bureau d'architecte et la forme qu'il prend lors de sa création au tournant du $\mathrm{XX}^{\mathrm{e}}$ siècle, dans la décennie suivante, au sortir de la Première Guerre mondiale, ainsi qu'à l'aube de la Seconde. Ces quatre périodes correspondent aux quatre associations majeures de Leclerc avec d'autres architectes. Entre chacune d'entre elles, il travaille seul, mais à chaque fois, il décide de fonder une nouvelle raison sociale, avec un nouvel associé.

Avant de nous arrêter sur chacun de ces quatre architectes et de voir leur rôle, ainsi que les tâches occupées au sein de l'atelier, il convient de donner quelques renseignements au sujet de la formation reçue par Leclerc. À côté de ses quatre associations, à maintes reprises, il travaille avec différents architectes, artistes ou artisans, sur des projets bien précis et lors de concours. Certaines collaborations et ses affiliations à diverses sociétés seront mentionnées, mais nous ne nous y attarderons pas.

Concernant le territoire géographique de ce cas d'étude, il est principalement genevois. En effet la majorité des protagonistes sont des ressortissants de ce canton ou y sont nés et la grande majorité de l'œuvre architectural de Leclerc s'y trouve. En conclusion, nous tâcherons de rendre compte de l'impact d'une association sur la production architecturale, ou du moins de formuler des hypothèses sur cette relation entre structure associative et œuvre construit.

\section{Formation}

Né le 2 septembre 1874 dans la commune de Plainpalais, Antoine Leclerc est le troisième d'une fratrie de huit enfants. Son père, Joseph Leclerc exerce le métier de droguiste avant de devenir Chancelier d'État en 1886, poste qu'il occupera près de vingt ans. Ce dernier aurait poussé son fils, quand il eut atteint l'âge de 15 ans, à se décider entre la profession de photographe ou celle d'architecte ${ }^{1}$. Le jeune homme, après

I. Beaucoup d'informations de type biographique proviennent de P. Nerfin, Antoine Leclerc (1874-1963), architecte à Genève. 
avoir fait son choix, suit brillamment durant une année les cours de la classe d'architecture dans l'une des écoles municipales d'art de Genève, remportant prix et accessit dans chaque discipline, sous la houlette du professeur Gédéon Dériaz. Peut-être ce dernier joue-t-il un rôle dans la décision prise par Joseph Leclerc d'envoyer son fils poursuivre ses études à Paris, Dériaz ayant été en son temps un des élèves les plus remarquables de l'École des beaux-arts. La préférence accordée à la capitale française plutôt qu'au Polytechnicum zurichois tient certainement également au fait que le parrain du jeune homme, Antoine Pouzait, y habite et peut donc l'assister dans les démarches administratives. Rappelons qu'à cette époque, bien que l'École des beaux-arts soit à son apogée, de plus en plus d'étudiants de Suisse romande se tournent vers Zurich, où le cursus d'études était plus court ${ }^{2}$.

Antoine Leclerc arrive donc à Paris en 1891 ; il a 16 ans et tous les matins, de 9 à 11 heures, il fréquente les cours de l'atelier préparatoire de Godefroye et Freynet, avant de travailler comme dessinateur les après-midis. Il était usuel depuis une dizaine année, que les futurs élèves architectes se forment avant leur admission aux Beaux-arts ${ }^{3}$. Au printemps 1893, Antoine Leclerc s'inscrit au concours d'entrée de la prestigieuse école. Pour son professeur Jules Godefroye, sa réussite ne fait aucun doute puisqu'il l'affirme dans une lettre envoyée au père du jeune homme:

non seulement votre fils sera reçu à cette session à l'école des beauxarts un des premiers mais encore il en sera par la suite un des plus brillants élèves. Il est du reste arrivé à Paris avec des données et une éducation artistique supérieure de beaucoup à celles de ses camarades, ce qui fait le plus grand honneur à ses professeurs de Genève ${ }^{4}$.

Le 3 août 1893, le verdict tombe. Antoine Pouzait, le parrain, envoie un télégramme à la famille Leclerc à Genève, ne contenant que trois mots: "Antoine reçu quinzième» 5 . Il fait partie de la même volée que

2. D. Lüthi, Le compas et le bistouri, p. 70-73.

3. L.-Th. David de Penanrun, L. F. Roux, E. A. Delaire, Les architectes élèves de l'école de Beaux-Arts, p. 121.

4. Lettre de Jule Godefroye à Joseph Leclerc, datée du 21 juin 1893, Archives d'État de Genève (AEG), Archives Privées 280.1.4.

5. Télégramme d'Antoine Pouzait à Joseph Leclerc, daté du 3 août 1893, AEG, cote générale Archives Privées 280, série 1. 
ses compatriotes genevois Alphonse Laverrière et Edmond Fatio ou que le français Louis Süe. Le rang d'admission offrant une certaine priorité sur le choix de l'atelier ${ }^{6}$, c'est tout naturellement que Leclerc choisit celui de Victor Laloux, atelier duquel était issu son ancien maître, Jules Godefroye. Au sein de l'atelier Laloux, au numéro 8 de la rue d'Assas, Leclerc rencontre celui qui deviendra son camarade de classe, son compagnon de voyage, mais surtout son indéfectible ami, Georges Gromort (1870-1961). Ce dernier, qui enseignera aux Beaux-arts, se fera connaître par ses nombreux ouvrages théoriques qui prennent la suite de ceux, non moins fameux, de Julien Guadet. Pour l'une de ses premières publications, certains relevés sont d'ailleurs signés de la main de Leclerc et ensemble les deux compères gagnent la troisième médaille au concours du Salon de l'exposition des Beaux-arts en 1900. Leur vie durant, ils entretiendront une correspondance épistolaire, éclairante à de multiples points de vue.

Leclerc est issu d'une famille que l'on peut certes qualifier d'aisée mais nombreuse. Afin de financer ses études, il ne peut donc compter sur le seul soutien de ses parents. Bien que bénéficiant à plusieurs reprises d'une bourse de la Fondation genevoise Lissignol, il doit travailler en parallèle pour des architectes parisiens, parfois dans des ateliers de fortune mal éclairés et installés dans des appartements (fig. 1). À la toute fin du XIX ${ }^{\mathrm{e}}$ siècle, Antoine Leclerc exécute les plans d'une luxueuse villa pour son travail de diplôme. Le 10 janvier 1900, il reçoit son diplôme officiel : dorénavant il est architecte, nanti du titre convoité et réputé de DPLG (diplômé par le gouvernement). Il a 25 ans et il est temps pour le jeune architecte de rentrer dans sa Genève natale après neuf ans passés dans la capitale française.

Lorsque Antoine Leclerc commence sa carrière au début du XXe siècle, il y a déjà sur le terrain genevois 47 agences d'architecture et 70 bureaux d'ingénieurs. En dix ans, le nombre des agences d'architectes passe à $80^{7}$. Au tournant du siècle, en Suisse romande, on constate un essor des ateliers d'architectes et de façon simultanée, un nombre croissant

6. L.-Th. David de Penanrun, L. F. Roux, E. A. Delaire, Les architectes élèves de l'école de Beaux-Arts, p. 114.

7. G. Barbey et al., «Genève», p. 272. 
Fig. 1 - Un appartement parisien de la fin du XIX siècle transformé en atelier d'architectes où a travaillé Antoine Leclerc.

d'association entre deux architectes ${ }^{8}$, peut-être à cause de l'ampleur que prennent les concours au même moment.

Ainsi, d'entrée de jeu, Leclerc ne déroge pas à la règle et s'allie avec deux personnes: son propre frère, Louis Leclerc, qui est ingénieur, et Gaston Le Cerf, un autre architecte. Si parfois, un jeune architecte s'associait avec un confrère plus âgé et donc expérimenté, ce n'est pas le cas de notre architecte.

\section{Leclerc \& Le Cerf-avenue de Frontenex 1}

En observant attentivement le cachet de leur agence (fig. 2), on remarque que c'est Antoine Leclerc qui domine: le titre DPLG, auquel il a rajouté un « $\mathrm{F}$ » pour insister qu'il est diplômé par le gouvernement français, s'étale sur toute la partie supérieure de l'en-tête. Il est vrai que ce titre était une plus-value incontestable aux yeux de la clientèle et donc à

8. D. Lüthi, La construction de l'architecte, p. 107. 


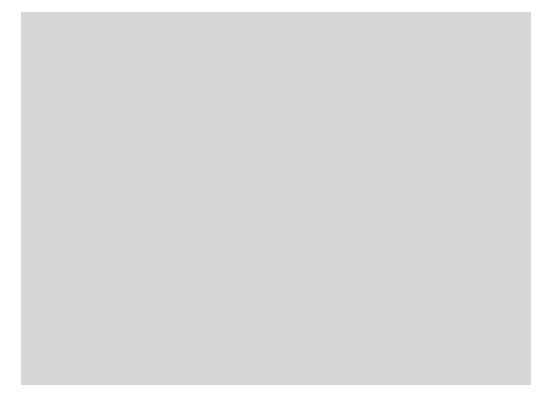

Fig. 2 - En-tête du bureau de Leclerc Frères et Le Cerf.

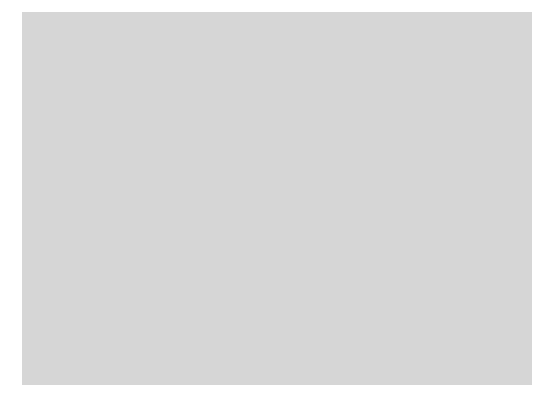

Fig. 3 - En-tête du papier à lettre d'Antoine Leclerc et Gaston Le Cerf.

valoriser. Parfois, ils signent "Leclerc Frères et Le Cerf». Louis Leclerc et Gaston Le Cerf sont de deux ans plus âgés que leur associé DPLG et les deux hommes sont également sapeurs-pompiers de la commune de Plainpalais. Gageons que c'est par ce biais qu'ils se sont rencontrés. Cependant le trio ne dure que peu de temps. Rivalité fraternelle ou tension houleuse due au caractère impétueux de Louis Leclerc - nous ne le saurons sans doute jamais - toujours est-il que ce dernier se retire de l'agence. Les deux associés restants conservent cependant le titre d'ingénieur, le mettant même au pluriel, comme si les deux l'étaient soudainement devenus (fig. 3), montrant surtout que la dénomination n'était pas encore protégée.

Gaston Le Cerf (1872-1936), avant de s'associer avec les frères Leclerc n'a pas conçu grand-chose. Fils d'un constructeur de bateau, de nationalité française mais né à Genève, il fréquente la classe d'architecture de l'École d'architecture et d'ornement de Genève, entre 1888 et 1889, époque où il n'y a que deux autres élèves architectes, Otto Carus et Alexandre Bordigoni, tout en travaillant à côté chez Bertrand \& Jaquet à la rue du Rhône $15^{\circ}$. Si l'historiographie locale a parfois mentionné son parcours à l'École des beaux-arts de Paris, le répertoire des anciens élèves de cette institution nous apprend qu'il n'en est rien. La possibilité qu'il l'ait fréquentée en qualité de simple auditeur ne peut cependant être totalement exclue.

9. A. Brulhart, "Les premiers pas d'Alphonse Laverrière», p. 26 et Registre de l'École d'architecture et d'ornement des années 1874-1896. Le Cerf est élève de cette école du 3 septembre 1888 au 22 juin 1889. 


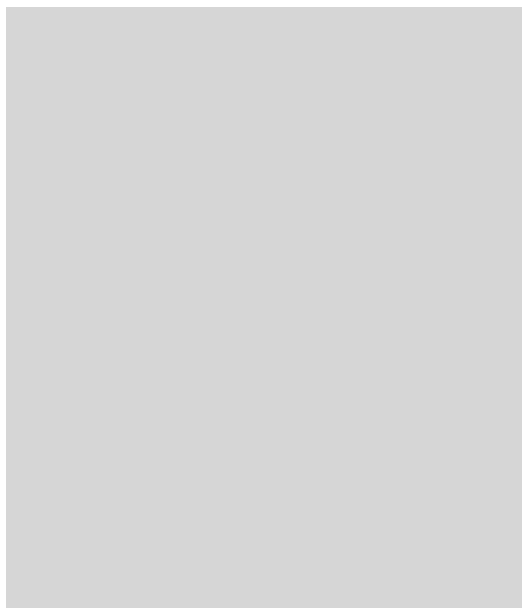

Fig. 4 - Tampon d'Henry Maillard accolé à celui de Leclerc et Le Cerf.
En 1898, Le Cerf milite activement pour la création d'une école technique à Genève (qui verra finalement le jour en 1901) et il est membre de la section genevoise de la Société des ingénieurs et architectes suisses (SIA) de 1903 à 1910 . Son retrait ne correspond pas à la fin de son association avec Leclerc survenue en 1907 et dont nous ignorons la raison; après cette date, plus aucune autorisation de construire n'est délivrée à son nom sur le territoire genevois. Le déménagement dans un autre canton serait une explication plausible, cependant il est domicilié en tout cas jusqu'en 1926 à Plainpalais dans un immeuble d'ailleurs construit avec Leclerc. L'année 1907 correspond à sa nomination en tant que premier lieutenant de la compagnie des sapeurs-pompiers de Plainpalais. Cependant, il réapparaît en 1912, en tant que conducteur de travaux, auprès de l'architecte Edmond Fatio sur le chantier de la Corraterie. Cette donnée plaide en faveur du rôle qu'il occupait dans le bureau Leclerc-Le Cerf, à savoir davantage occupé par les chantiers, coordonnant les différents corps de métier.

Avec Antoine Leclerc, Gaston Le Cerf aura participé à la construction de 22 immeubles de rapport, de 7 villas et d'une petite dizaine d'autres bâtiments. En 1903, leur bureau se joint à celui de l'architecte Henry Maillard (1873-....) pour réaliser tout un îlot d'immeubles à Plainpalais sur les anciennes terres de l'Exposition nationale de 1896 (fig. 4).

Comme il s'agissait d'une importante opération immobilière, nous pouvons supposer que cette union temporaire, ce "consortium» si l'on peut dire, permettait de développer une force de travail plus grande, et plus rassurante aux yeux des commanditaires. Sur Henry Maillard, né à Genève bien que d'origine vaudoise, nous ne savons ainsi dire que peu de choses, mis à par le métier qu'exerçait son père, cuisinier. D'après le registre de l'École d'architecture et d'ornement des années 1874-1896, Henry Maillard y aurait été brièvement élève du 2 mars 1891 au 20 juin 


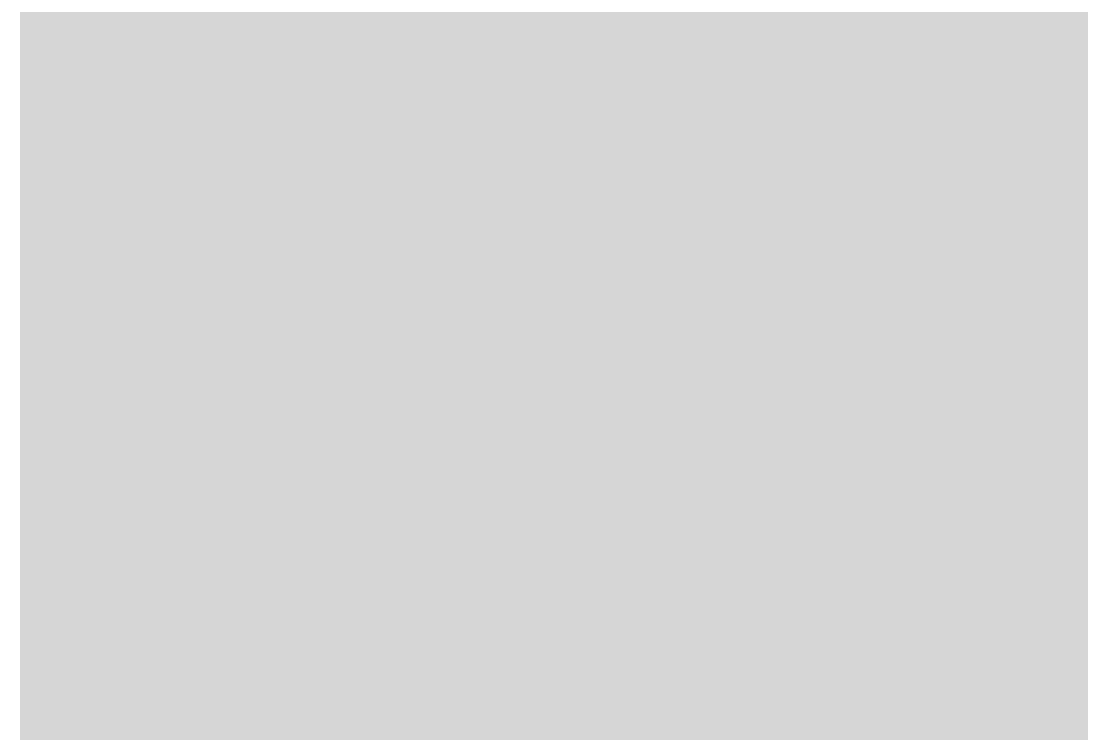

Fig. 5 - Lettre d'annonce d'ouverture du bureau Leclerc et Gambini.

1891 et travaillait alors chez l'architecte Gonthier; c'est tout ce que nous savons.

\section{Leclerc \& Gambini - avenue de Lancy 13}

Après la dissolution de son bureau avec Le Cerf en 1907, Leclerc travaille seul jusqu'en 1910. En décembre de cette année-là, il s'associe avec Charles Auguste Gambini, un autre architecte, mais non "DPLGF", comme le distingue nettement leur papeterie. Leur bureau est logé au sein d'un immeuble construit par les frères Leclerc et Le Cerf en 1901, à l'avenue de Lancy 13 (actuellement avenue Henri-Dunant 16).

Grâce à une lettre d'information qui annonce leur union et la reprise des affaires du bureau de Leclerc à leurs clients ou potentiels maîtres d'ouvrages (fig. 5), nous en apprenons davantage sur la nature de leurs activités : établissement d'avant-projets et de plans d'exécutions pour tous genres de constructions, relevés, devis, direction et surveillance des travaux, vérification des mémoires d'entrepreneurs, décoration et 


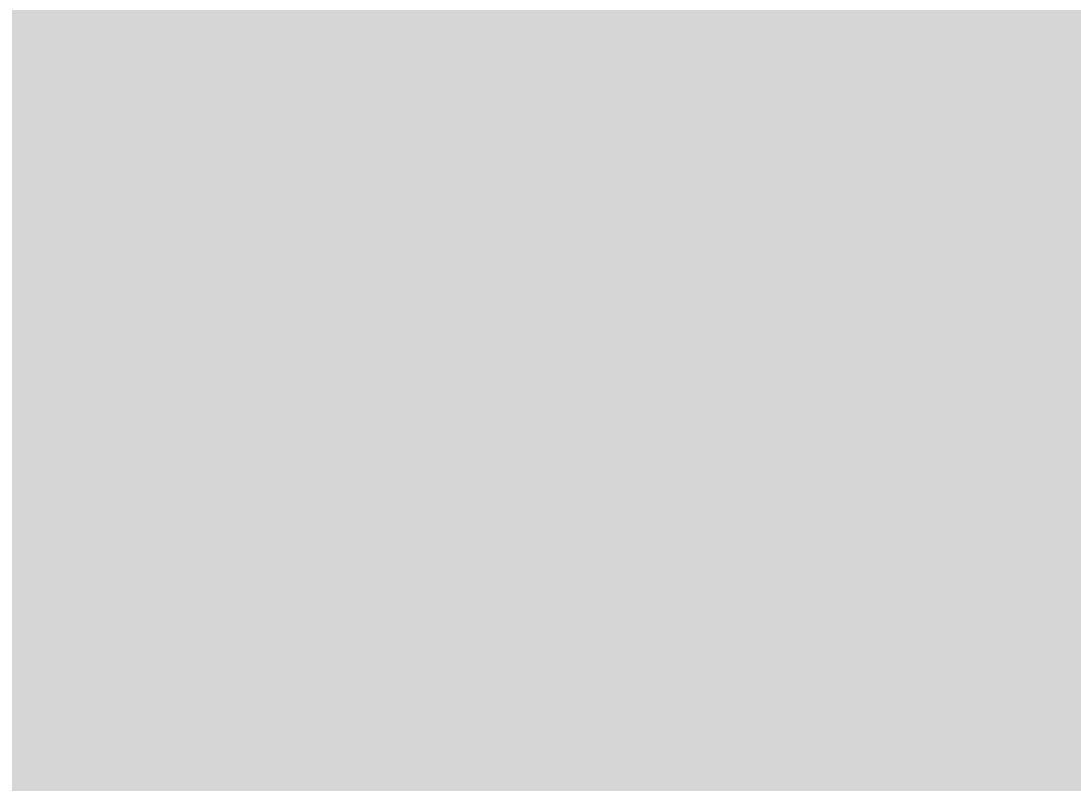

Fig. 6 - Liste des réalisations de Leclerc et Gambini. • = Leclerc.

aménagement de mobilier, expertises et arbitrages, études de lotissements de terrains et placements hypothécaires. Avec la lettre, une liste des productions architecturales des deux jeunes associés était livrée (fig. 6). On peut y constater que Gambini a encore très peu construit. Pourtant il avait, à quelques jours près, le même âge que Leclerc. Citons l'Hôtel moderne à Evian-les-Bains et un aménagement de combles pour Louise Démole, qui est en fait sa propre mère. Elle sera d'ailleurs l'une des premières clientes du nouveau bureau, commandant un immeuble de rapport. Quant au père de Gambini, fils du Consul d'Italie, il s'agit d'un pasteur quelque peu connu, ayant professé à Berlin avant de revenir s'installer à Genève. Nous pouvons formuler l'hypothèse que c'est par son biais, autant que par ses propres qualités de fidèle paroissien, qu'Antoine Leclerc devient en 1911 le secrétaire adjoint des biens curiaux du Consistoire de l'église nationale protestante de Genève, ainsi que le préposé à l'entretien, s'assurant ainsi un nombre de commandes non négligeable.

Si nous ignorons tout de la rencontre entre Leclerc et Gambini, nous savons que leur première collaboration a lieu autour d'un chalet, celui 


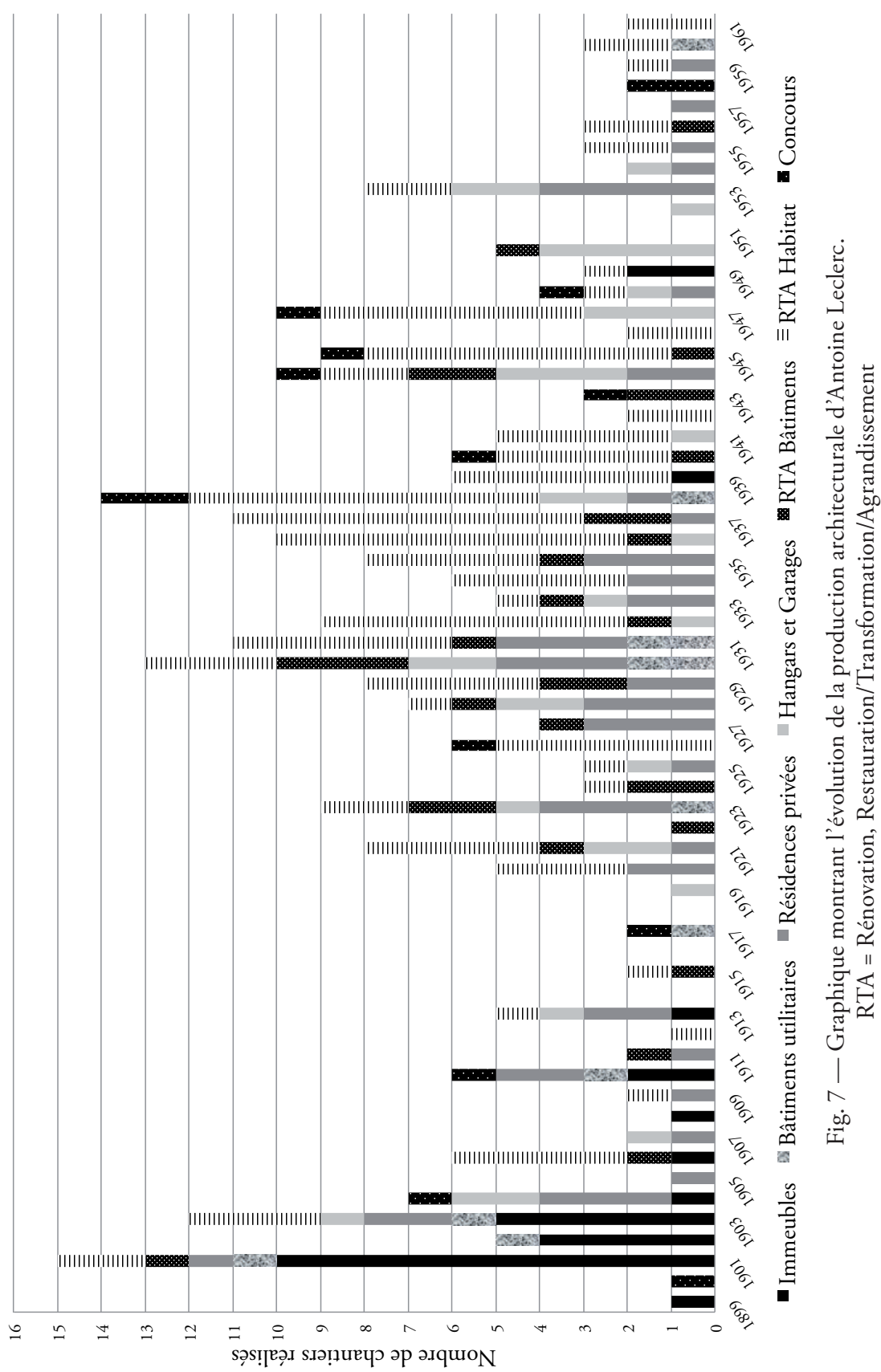


de Marc Chambaud, à Corbeyrier-sur-Aigle, en terres vaudoises. En effet, sur les plans de ce chalet ${ }^{10}$, les deux tampons, encore distincts, des deux architectes cohabitent. Cette expérience commune positive les a sans doute poussés à s'associer. Après plusieurs immeubles, villas et chalets conçus ensemble, la construction de la salle de paroisse protestante de Carouge, ainsi que la restauration et transformation du temple de la Fusterie, Leclerc et Gambini se séparent en 1914. La date n'est certainement pas due au hasard: avec le début de la guerre (fig. 7), les commandes et les chantiers se raréfient, entraînant possiblement la dissolution de l'agence.

Pendant les années du conflit, Leclerc pourvoit à quelques petits chantiers et surtout à sa plus grande commande publique, l'école du Mail, pour la commune de Plainpalais. Quant à Charles Auguste Gambini, il ne construit apparemment rien jusqu'en 1920, date à laquelle il signe un groupe de cinq immeubles avec l'entrepreneur Ernest Dumont. En 1924, il dessine quelques annexes de bâtiments, puis nous perdons sa trace. Le fait qu'il vienne d'une famille bourgeoise explique peut-être d'autres sources de revenus lui ayant permis de délaisser sa profession d'architecte.

Au début de leur association, Leclerc et Gambini s'adjoignent les services d'Adolphe Guyonnet (1877-1955), le futur architecte du célèbre Pavillon de la Conférence du désarmement (1932) pour participer au concours pour l'Hôtel de la Caisse d'épargne de Genève. Leur projet, "Suk» décroche le $5^{\mathrm{e}}$ prix ${ }^{11}$. Tout comme Leclerc, mais à l'inverse de Gambini, Guyonnet était membre de la SIA et de la FAS, la Fédération suisse des architectes. S'ils ne se sont pas connus aux Beaux-arts de Paris, les deux architectes se sont probablement croisés à des séances de ces sociétés. Plus tard, Antoine Leclerc enverra sa fille, Marie-Louise, réaliser un stage dans l'agence de son confrère Guyonnet.

\section{Camoletti et Leclerc - boulevard Georges-Favon 24}

Au début de l'année 1920, Leclerc déménage son bureau, rejoignant celui d'Alexandre Camoletti au boulevard Georges-Favon 24. Il est difficile de

Io. AEG, cote Archives Privées 280.5.8 et 280.5.9 (dossier chalet Chambaud).

II. "Concours pour l'Hôtel de la Caisse d'épargne, à Genève». 
constater une association officielle à proprement parler, cependant dans les annuaires ou sur certains plans signés à deux, nous pouvons lire la dénomination suivante: «bureau d'architectes, Alexandre Camoletti et Antoine Leclerc». On constate que pour la première fois, Leclerc n'occupe pas la position de tête. Camoletti était également issu de l'École des beaux-arts. Admis une année après Leclerc, en 1894, il a été l'élève d'Henri Deglane ${ }^{12}$. Les deux Genevois ont probablement sympathisé lors de leur séjour parisien; ils suivent le cursus complet, deuxième et première classe et obtiennent tous deux leur diplôme en 1900. Avant de partir pour Paris, Alexandre, neveu des architectes John et Marc fait son apprentissage chez Louis-Etienne Poncy - lui-même formé par John Camoletti ${ }^{13}$ - avant de poursuivre sa formation chez son oncle Marc Camoletti.

Alexandre Camoletti est connu pour avoir été professeur, tant à l'École des beaux-arts de Genève qu'à celle des arts et métiers. Patriote, il lutte contre l'«enlaidissement de Genève» ${ }^{14}$. L'architecte participe à un nombre élevé de concours, souvent en collaboration avec d'autres confrères. En 1906, il remporte avec Henry Baudin le $4^{\mathrm{e}}$ prix pour le collège de Nyon et ils se voient attribuer le mandat d'exécution, ce qui n'est pas sans déclencher une certaine polémique, puisque la coutume voulait que le projet lauréat soit réalisé ${ }^{15}$. On lui connait, par la suite, une association de quatre ans avec Alfred Olivet (1863-1942), architecte avec lequel il réalise l'école de Saint-Jean en 1912, l'Institut d'Hygiène de Genève en 1915. Ensemble, ils dessinent un projet pour le Museum d'histoire naturelle, ainsi que pour le Pont Butin. Camille Martin écrira en hommage à Camoletti: "cet homme qui avait des idées, dut le plus souvent travailler pour ceux qui n'en avaient pas" ${ }^{16}$. Seul, Camoletti

I2. L.-Th. David de Penanrun, L. F. Roux, E. A. Delaire, Les architectes élèves de l'école de Beaux-Arts, p. 203.

13. D. Ripoll, Musée d'ethnographie (ancienne école primaire), Bd Carl-Vogt 65-67Genève, p. 16.

I4. F. F., «Nécrologie, Alexandre Camoletti», p. 53: «Passionné par la beauté, et profondément attaché à son pays, il souffrait de le voir défiguré par les emprises de la spéculation, et sortait de sa réserve ordinaire afin de mener campagne pour l'en protéger."

15. "Alexandre Camoletti" et M. Jacquet, "Concours et controverses pour deux écoles urbaines", p. 158.

I6. C. Martin, «Alexandre Camoletti». 
signe les plans de la Maison du Faubourg de Saint-Gervais érigée entre 1916 et $1919^{17}$.

Sur sa collaboration avec Leclerc, il n'est pas aisé de se faire une idée. Chacun d'eux semble vaquer à ses propres projets, tout en secondant l'autre selon les besoins du moment. En effet, des autorisations de construire sont délivrées à l'un ou l'autre, rarement aux deux ensemble ${ }^{18}$. Cependant, il arrive que Leclerc signe des lettres ou des rapports à la place de Camoletti et vice-versa. Si l'aspect administratif semble partagé, la conception de projets architecturaux communs reste pour l'heure impossible à prouver.

Gravement malade, ce dernier meurt en 1923 et Leclerc hérite de tout son mobilier de bureau. Les tables à dessin aussi bien que les équerres ou les tés étaient pyrogravés des initiales A.C. que Leclerc gardera avec affection toute sa vie. Il continue de travailler à la même adresse jusqu'en 1934.

\section{Leclerc \& Cie - rue de l'Université 7}

La dernière personne avec qui Antoine Leclerc s'associe n'est autre que sa propre fille, Marie-Louise Leclerc (1911-2001). Elle reçoit son diplôme de dessinatrice-architecte à l'École des beaux-arts de Genève en 1936, ce qui fait d'elle la première femme architecte de Suisse romande ${ }^{19}$. Après avoir effectué un stage chez Guyonnet, Marie-Louise Leclerc entreprend un voyage d'études en Italie, empruntant les traces de son père, trentesept ans auparavant. Dès son retour, elle commence à travailler à ses côtés. Parallèlement, elle obtient des commandes en tant que dessinatrice: elle réalise des illustrations et des peintures murales et conçoit des affiches et des vitraux. Ce n'est qu'en 1942 qu'elle devient officiellement l'associée de son père, comme en témoigne l'inscription au registre du commerce: "Il est constitué une société en nom collectif, ayant pour objet l'exploitation d'un bureau d'architecte, et branches annexes, et la

17. B. Chaillot-Calame, Maison du Faubourg, Rue des Terreaux-du-Temple 6-8, Saint-Gervais, Genève.

I8. L'autorisation de construire pour la maison de M. Vernier est par exemple adressée aux deux architectes, AEG, TP 1923/99 et TP 1923/350.

19. É. Lang, Les premières femmes architectes de Suisse et AEG, Archives Privées 280.2.2 et 280.2.3, correspondance entre Évelyne Lang et Marie-Louise Leclerc. 
reprise des activités du bureau dont Monsieur Leclerc est actuellement titulaire ${ }^{20}$. Cependant, Marie-Louise Leclerc n'hésite pas à s'engager sur des projets extérieurs au bureau, par exemple en collaborant avec une autre femme architecte, Anne Torcapel, également fille d'architecte. Les deux pionnières transforment et rénovent la Taverne de la Madeleine, agrandissent la maternité de Genève et construisent la policlinique gynécologique, ainsi que le bloc opératoire de la maternité ${ }^{21}$. Avec son père, Marie-Louise gagne plusieurs prix lors de participations à des concours, mais ils n'obtiennent jamais de réalisation. On peut citer le concours pour la banque valaisanne en 1938 ( $3^{\mathrm{e}} \mathrm{rang}$ ), celui du pavillon GallandAsile de Vessy la même année ( $2^{\mathrm{e}}$ prix) ${ }^{22}$ ou encore pour l'hôpital de Sion en 1939 ( $3^{\mathrm{e}}$ prix). Si Antoine Leclerc a de tout temps répondu à des concours, nul doute que l'arrivée en renfort de sa fille a relancé son enthousiasme à y participer plus régulièrement. En 1961, Leclerc \& Cie est contraint à chercher de nouveaux locaux, car l'immeuble sis rue de l'Université 7 est voué à la démolition. Finalement, père et fille décident de retourner à l'avenue Henri-Dunant 16, immeuble que Leclerc avait construit en 1901 et où il avait déjà eu son bureau avec Gambini.

En février 1963, très âgé, Antoine Leclerc s'arrête de travailler et la raison sociale Leclerc $\&$ Cie est radiée. Il décède le $1^{\text {er }}$ décembre de la même année. Marie-Louise reprend la suite des affaires: elle s'occupe de l'entretien et de réfections des constructions de son père, signe quelques maisons et des transformations jusqu'à la fin des années 1980, puis elle prend sa retraite.

\section{Collaborations ponctuelles et fonctionnement}

En dehors des quatre associations que nous venons de décrire, Antoine Leclerc a travaillé de temps à autre avec d'autres architectes. Citons Antoine Céria (1881-1967) avec lequel il modifie plusieurs intérieurs dans les années 1930, Edmond Fatio (1871-1959) avec qui il transforme un immeuble de la Vieille-Ville, ainsi qu'Adrien Haas (18741960), Auguste Bordier (1875-1965) et Émile-Albert Favre (...-1947)

20. AEG, Archives Privées 280. 4.2.

2I. AEG, Fonds Leclerc, entrée 2004/030, n³ 30, non inventorié.

22. " "Pavillon Galland" im Altersasyl Vessy (Genf)». 
avec lesquels il semble collaborer sur le chantier du temple de Champel entre 1931 et 1933, bien que tous les plans portent uniquement son tampon. Mentionnons encore le chantier de transformation du temple de Carouge en 1923 où il collabore avec le peintre et décorateur Erich Hermès (1881-1971) et la réalisation de la villa L'Escale Fleurie en 1932 lorsqu'il coopère avec le décorateur et ensemblier Percival Pernet (18901977).

Concernant l'organisation de ses bureaux, nous n'avons trouvé presque aucune mention d'employés subalternes, ce qui laisse envisager qu'il s'agissait, lors de toutes ses associations, d'agences de petites dimensions. Bien sûr, l'immense majorité des papiers de bureau n'existant plus ou ayant disparu, il n'est pas possible de le confirmer ${ }^{23}$. Une petite occurrence de main-d'œuvre est cependant constatée: en 1950, Leclerc et Cie paie la somme de 200 francs pour un employé extérieur, un dessinateur. Sans d'autres preuves matérielles, nous ne pouvons estimer dans quelle mesure Leclerc a sous-traité une partie du travail des plans de façon régulière. Lorsqu'il décède, un certain $\mathrm{C}$. Cavallin, architecte établi à Cannes, écrit une lettre de condoléances à Marie-Louise où il fait l'éloge de celui qui lui a tout appris, de son «ancien patron»:

Cette disparition me rappelle tout un passé, un passé de jeunesse et aussi d'étude, car si aujourd'hui à 64 ans j'exerce la même profession, je le dois à celui qui m’a guidé le premier, qui m’a conseillé, qui m’a fait aimer ce métier. Les qualités d'artiste et la droiture de votre père ont toujours été pour moi un exemple et une conduite $[\ldots]^{24}$.

Il est ainsi possible d'envisager la présence de ce Cavallin au sein de l'un des bureaux d'Antoine Leclerc, en tant qu'apprenti ou stagiaire, une quarantaine d'années auparavant.

De manière générale, lors de ses principales associations, s'il semble ardu d'esquisser un partage des tâches au sein de chaque bureau, de chaque atelier, Antoine Leclerc semble émerger à chaque fois, excepté

23. Les seules traces de comptabilité qui subsistent sont celles de la dernière période d'activité du bureau Leclerc et Cie, qui nous renseigne entre autres sur le prix du loyer des locaux et les salaires. AEG, Archives Privées 280.4.3 à 280.4.9.

24. Lettre de C. Cavallin à Marie-Louise Leclerc, datée du 29 février 1964, AEG, Fonds Leclerc, entrée 2006/004, non inventorié. 
avec Camoletti, comme l'élément inventif, créateur, correspondant à la figure de l'architecte-artiste. Le côté gestionnaire, commercial, ne semble guère l'intéresser, même s'il est à maintes reprises forcé d'endosser le double statut, notamment lorsqu'il travaille seul. Il paraît cependant relativement à l'aise avec l'administratif, n'hésitant pas à user de sa verve, teintée parfois d'un soupçon d'ironie, lorsqu'il envoie des lettres pour contester les décisions du Département des travaux publics qu'il juge injustifiées ou incohérentes.

On connaît le fonctionnement de la grande agence américaine d'Adler et Sullivan, où le premier s'occupe de la clientèle tandis que le second se concentre sur la création. Éloignés du modèle anglo-saxon par sa taille et par son fonctionnement, les différents bureaux de Leclerc témoignent d'une plus grande polyvalence. Sans entrer dans une étude approfondie de leur clientèle, nous savons que les maîtres d'ouvrage les plus prestigieux de Leclerc lui sont envoyés par son frère Frédéric, qui était banquier. Le réseau familial et paroissial, voire de simple voisinage, était également un important pourvoyeur de commandes.

\section{Société(s)}

Après avoir évoqué les sociétés et associations d'architectes créées par Antoine Leclerc, passons en revue les sociétés auxquelles il était affilié. Fort de son titre d'architecte DPLG, Leclerc est appelé à siéger dans divers organismes. Il fait partie d'une société régionale française d'architectes, comme beaucoup d'anciens étudiants des beaux-arts. Très rapidement après son installation, en 1901, il adhère à la SIA (Société des ingénieurs et architectes suisses), dont il entre au Comité de la section genevoise en 1921, alors présidée par Edmond Fatio. En 1906, avec Henry Baudin, architecte connu en Suisse pour ses nombreuses publications, il rédige l'album de l'assemblée générale suisse de la SIA. En 1927, il intègre l'Association Syndicale des Architectes pratiquants du Canton de Genève (ASA) et devient également membre de la FAS (Fédération suisse des architectes). Nous ne savons donc pas à quelle délégation il est rattaché en 1953 lorsqu'une commission de douze architectes est chargée d'étudier «la voie à suivre pour arriver à une solution rapide et concrète 
du problème de la reconstruction du Grand Théâtre » ${ }^{25}$. La commission est en fait composée de quatre délégués pour chaque association majeure d'architectes de la place, soit SIA, AGA (ancienne ASA), FAS et CIAM. Ce groupe d'experts comprend également Paul Waltenspühl, Marc-Joseph Saugey ou Albert Cingria pour ne citer que les plus célèbres d'entre eux.

La place qu'occupe Antoine Leclerc dans la société, qu'elle soit civile ou institutionnelle, dénote une grande implication. Discret, il fait un peu de politique sous les couleurs démocrates au sein de sa commune de Carouge. Leclerc demeure des années durant dans la commission de surveillance de l'École des beaux-arts de Genève et à de nombreuses reprises il est appelé à siéger dans divers jurys. Que cela soit Saugey lorsqu'il signe sa nécrologie ${ }^{26}$ ou d'autres sources plus disparates, tous s'accordent sur le sérieux et la grande humilité qui caractérisaient notre architecte. Nous l'avons vu, des traces de ses multiples engagements subsistent, mais il reste hasardeux d'en déduire une stratégie particulière. Néanmoins, et malgré la reconnaissance par ses pairs de son vivant, Antoine Leclerc n'échappe pas au destin de beaucoup de ses confrères du début du $\mathrm{XX}^{\mathrm{e}}$ siècle: il tombe dans l'oubli.

\section{En guise de conclusion}

Sur une carrière aussi longue, plus d'un demi-siècle riche en évènements historiques et politiques, il est délicat de tirer des conclusions quant à l'influence des collaborations précitées sur la production architecturale de Leclerc. L'impact le plus significatif relève sans doute davantage de la typologie des constructions (fig. 7). Si près des deux tiers de l'œuvre bâti de Leclerc concerne l'habitat en général, l'écrasante majorité des immeubles de rapport sort de terre lors de son association avec Le Cerf (22 immeubles sur un total de 29). Ce chiffre peut s'expliquer par une démographie décroissante pendant plusieurs décennies après la Grande Guerre, donc par une baisse de la demande pour la construction de logements. Lorsque Leclerc travaille seul, il ne dirige jamais le chantier d'un

25. Archives de la Ville de Genève (AVG), Mémorial du Conseil municipal de la Ville de Genève, n 3, 29 juin 1954, p. 187.

26. M.-J. Saugey, «Totentafel, Antoine Leclerc, architecte FAS». 
immeuble, préférant dessiner des résidences privées. Au total il signe les plans de plus de 60 villas, maisons et chalets en tout genre. Leclerc conçoit également une cinquantaine de bâtiments utilitaires, privés comme publics et conduit plus de 160 interventions sur du bâti existant. Le volume de sa production, près de 300 chantiers, autorise à le qualifier d'architecte prolifique. Ses participations à des concours, plus d'une vingtaine, sont disséminées tout le long de sa carrière. Au niveau de la réception et de la critique, les articles qui sont consacrés à certaines de ses réalisations datent de ses débuts, la plus grande proportion concerne sa période d'union à Le Cerf. Quant au style à proprement parler, nous pouvons définir Antoine Leclerc comme un architecte sachant s'adapter au désir de ses commanditaires, comme aux changements de contexte (à la fois social et historique) et réagir à la modernité. La «touche personnelle», certaines petites marottes typiques de Leclerc, apparaissent d'un projet à l'autre, mais la part exacte, l'apport «artistique» de ses différents associés reste pour l'heure, malgré le désir de le définir, encore nébuleux.

Pauline Nerfin

Université de Genève 


\section{BIBLIOGRAPHIE}

«Alexandre Camoletti», Das Werk, 10, 8 (1923), p. 209.

"Concours pour l'Hôtel de la Caisse d'épargne, à Genève", Bulletin technique de la Suisse romande, 36, 4 (1910), p. 41-42 et 36, 8 (1910), p. 93.

" "Pavillon Galland” im Altersasyl Vessy (Genf)", Schweizerische Bauzeitung, 111-112, 26 (1938), p. 327.

Barbey, Gilles et al., "Genève", in Inventaire suisse d'architecture 18501920, éd. par Hanspeter Rebsamen, Peter Röllin, Berne, Société d'Histoire de l'Art en Suisse, 1984, vol. 4, p. 272.

Brulhart, Armand, "Les premiers pas d'Alphonse Laverrière", in Alphonse Laverrière, 1872-1954: parcours dans les archives d'un architecte, éd. par Pierre Frey, Lausanne, Presses polytechniques et universitaires romandes, 1999, p. 26.

Chaillot-Calame, Babina, Maison du Faubourg, Rue des Terreaux-duTemple 6-8, Saint-Gervais, Genève, Conservation du patrimoine architectural de la Ville de Genève, rapport historique non publié, 1997.

David de Penanrun, Louis-Thérèse, Roux, Louis François, Delaire, Edmond Augustin, Les architectes élèves de l'école de Beaux-Arts: 1793-1907, Librairie de la Construction moderne, Paris, 1907 ( $2^{\mathrm{e}}$ édition).

F. F., «Nécrologie, Alexandre Camoletti », Schweizerische Bauzeitung, 82, 4 (1923), p. 53.

Jacquet, Martine, "Concours et controverses pour deux écoles urbaines", in Alphonse Laverrière, 1872-1954: parcours dans les archives d'un architecte, éd. par Pierre Frey, Lausanne, Presses polytechniques et universitaires romandes, 1999, p. 158-160.

LANG, Évelyne, Les premières femmes architectes de Suisse, thèse de doctorat, École polytechnique fédérale de Lausanne, 1992.

Lüthi, Dave, La construction de l'architecte. Histoire d'une profession en Suisse romande, 1800-1940, Éd. Alphil, Presses universitaires suisses, Neuchâtel, 2010. 
-, Le compas \& le bistouri. Architectures de la médecine et du tourisme curatif: l'exemple vaudois (1760-1940), Lausanne, BHMS, 2012.

Martin, Camille, "Alexandre Camoletti», Das Werk, 11, 4 (1924), p. 110-111.

Nerfin, Pauline, Antoine Leclerc (1874-1963), architecte à Genève, mémoire de maîtrise, Université de Genève, août 2014.

Ripoll, David, Musée d'ethnographie (ancienne école primaire), Bd CarlVogt 65-67-Genève, Conservation du patrimoine architectural de la Ville de Genève, étude historique non publiée, juillet 2000. Saugey, Marc-Joseph, "Totentafel, Antoine Leclerc, architecte FAS", Das Werk, 51, 2 (1964), p. 35.

\section{Crédits iconographiques:}

Fig. 1-3: AEG, Fonds Leclerc.

Fig. 4: AEG, dossier d'autorisation de construire TP 1903/Travaux A 120 Annexe 308.

Fig. 5-6: AEG, AP 280.4.1. 